\title{
Comparison of propagation- and grating-based x-ray phase-contrast imaging techniques with a liquid-metal-jet source
}

\author{
T. Zhou *a , U. Lundström ${ }^{\mathrm{a}}$, T. Thüring ${ }^{\mathrm{b}, \mathrm{c}}$, S. Rutishauser ${ }^{\mathrm{b}, \mathrm{c}}$, D. H. Larsson ${ }^{\mathrm{a}}$, M. Stampanoni ${ }^{\mathrm{b}, \mathrm{c}}$, \\ C. David ${ }^{\mathrm{b}}$, H. M. Hertz ${ }^{\mathrm{a}}$, and A. Burvall ${ }^{\mathrm{a}}$

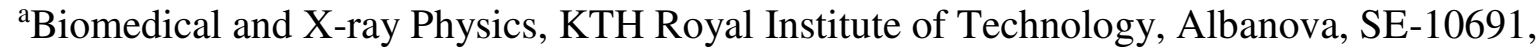 \\ Stockholm, Sweden; ${ }^{b}$ Paul Scherrer Institut, Villigen PSI, Switzerland; ' Institute for Biomedical \\ Engineering, Swiss Federal Institute of Technology, Zurich, Switzerland
}

\begin{abstract}
$\mathrm{X}$-ray phase-contrast imaging has been developed as an alternative to conventional absorption imaging, partly for its dose advantage over absorption imaging at high resolution. Grating-based imaging (GBI) and propagation-based imaging (PBI) are two phase-contrast techniques used with polychromatic laboratory sources. We compare the two methods by experiments and simulations with respect to required dose. A simulation method based on the projection approximation is designed and verified with experiments. A comparison based on simulations of the doses required for detection of an object with respect to its diameter is presented, showing that for monochromatic radiation, there is a dose advantage for PBI for small features but an advantage for GBI at larger features. However, GBI suffers more from the introduction of polychromatic radiation, in this case so much that PBI gives lower dose for all investigated feature sizes. Furthermore, we present and compare experimental images of biomedical samples. While those support the dose advantage of PBI, they also highlight the GBI advantage of quantitative reconstruction of multimaterial samples. For all experiments a liquid-metal-jet source was used. Liquid-metal-jet sources are a promising option for laboratory-based phase-contrast imaging due to the relatively high brightness and small spot size.
\end{abstract}

Keywords: X-ray imaging, phase-contrast, propagation-based, grating-based

\section{INTRODUCTION}

\subsection{Phase-contrast methods}

Conventional x-ray imaging registers the attenuation of the intensity after passage through an object. This attenuation is primarily caused by absorption in the object, and usually called absorption imaging. Phase-contrast imaging, on the other hand, utilizes the phase information which can be orders of magnitudes more sensitive than absorption information for some materials such as soft tissue. There are different methods of phase-contrast imaging, among which the propagationand the grating-based methods are discussed and compared here. PBI utilizes the propagation of the wavefront in free space and obtains the phase information as the edge enhancements [1]. GBI uses a Talbot grating interferometer and captures a series of images when the two gratings are at different relative positions of each other [2]. Source gratings are used when using an extended source [3]. The variance of the intensities of each pixel can be approximately analyzed with Fourier series, from which absorption, phase and scattering information can be obtained simultaneously [4].

Image quality has been studied in different ways for GBI differential phase contrast (DPC) images [5-13]. Comparisons with absorption imaging $[14,15]$ show that GBI and PBI give higher SNR at higher spatial resolution while absorption imaging gives higher SNR at lower spatial resolution. Here we compare PBI and GBI to each other in a direct way with respect to dose [16], which is an important issue for medical imaging, to investigate the detectability of the two methods for different object feature sizes.

\subsection{Liquid-metal-jet source}

PBI and GBI both have the advantages of robust experimental arrangements and good compatibility with polychromatic sources, which are needed for more practical applications. If aiming at small-animal imaging with high resolution, a microfocus source would be needed. For the sake of better dose efficiency, a larger source-to-object distance would be preferred, if keeping the same magnification. For a microfocus source which has relatively low flux compared to other sources such as synchrotron radiation sources, a larger distance can lead to a much longer exposure time to obtain good

Medical Imaging 2014: Physics of Medical Imaging, edited by Bruce R. Whiting, Christoph Hoeschen, Despina Kontos, Proc. of SPIE Vol. 9033, 903353 - (c) 2014 SPIE · CCC code: 1605-7422/14/\$18 · doi: 10.1117/12.2043417 
enough images. We use a liquid-metal-jet source in the experiments as its flux is higher than that of a normal microfocus source [17]. Liquid-metal-jet sources use regenerative liquid alloy jets as anodes, which avoids the risk of melting the anode under high e-beam power. In the present experiments the source was operated at a power density of around $2000 \mathrm{~kW} / \mathrm{mm}^{2}$.

\section{METHODS}

\subsection{Experiments}

Experiments were done with a galinstan liquid-metal-jet source with a spot size (FWHM) of $3.6 \mu \mathrm{m} \times 6.8 \mu \mathrm{m}$, which gives sufficient coherence length for GBI to use a Talbot interferometer which contains a phase grating and an absorption grating. The specifications of the gratings are given in Table 1.

Table 1. Grating specifics

\begin{tabular}{|c|c|c|c|c|c|}
\hline & Material & Thickness $(\mu \mathrm{m})$ & Substrate material & Substrate thickness $(\mu \mathrm{m})$ & Period $(\mu \mathrm{m})$ \\
\hline G1 & $\mathrm{Ni}$ & $10-11$ & $\mathrm{Ti}$ & 50 & 4.12 \\
\hline G2 & $\mathrm{Au}$ & 60 & $\mathrm{Si}$ & 500 & 2.4 \\
\hline
\end{tabular}

According to Raupach et al [15], for GBI the optimal performance for the phase image compared to the absorption image occurs when the beam separation $s$ equals the coherence length $\xi$, where $s=2 \lambda d_{2} / p_{1}$ and $\xi=d_{1} \lambda / 2 \pi \sigma$, in which $\lambda$ is the wavelength, $d_{1}$ and $d_{2}$ are the source-to-object distance and object-to-detector distance, respectively, $p_{1}$ is the period of G1, and $\sigma$ describes the width of the source spot if assuming a Gaussian distribution. If using the wavelength corresponding to the designed energy $25 \mathrm{keV}$ and the source size in the direction perpendicular to the grating pillars, we can get that the best $d_{2}$ for the $d_{1}$ we have now should be between $3^{\text {rd }}$ and $5^{\text {th }}$ fractional Talbot distance. 3rd was used in experiments. For PBI, the objects were kept at the same distance as for GBI. Then the dose is the same for both methods, assuming the same exposure time, while coherence properties are also similar. The propagation distance was limited by the field of view of the detector. The experimental arrangements are shown in Figure 1 and listed as distances from the source in Table 2.

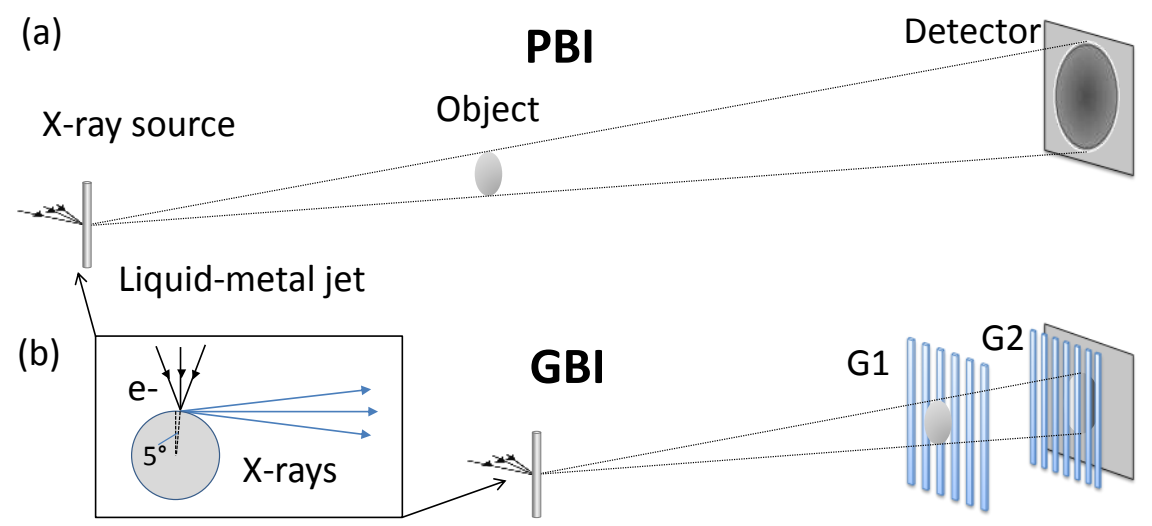

Figure 1. Experimental arrangements for (a) PBI and (b) GBI.

Table 2. Experimental geometry parameters.

\begin{tabular}{|c|c|c|c|c|}
\hline Distances $(\boldsymbol{m})$ & Object & G1 & G2 & Detector \\
\hline PBI & & - & - & 2.188 \\
\hline GBI & 0.874 & 0.903 & 1.052 & 1.091 \\
\hline
\end{tabular}

The detector used in the experiments is a scintillator-based CCD camera with a pixel size of $9 \mu \mathrm{m} \times 9 \mu \mathrm{m}$, and a PSF with a measured FWHM of about $21 \mu \mathrm{m}$. The first object is a polyethylene terephthalate (PET) monofilament phantom. The second object is a breast biopsy sample fixed with formalin in cylindrical sample tube with diameter of about $1 \mathrm{~cm}$. The third object is a rat brain embedded in paraffin. Objects were immersed in liquid paraffin during exposure to avoid 
phase-wrapping. PBI has no problem of phase-wrapping, but the bath was still used to keep the spectrum and dose the same.

\subsection{Simulations}

To compare the two methods quantitatively, the signal-to-noise ratio (SNR) is calculated assuming an ideal observer in an SKE/BKE (signal-know-exactly/background-know-exactly) task in Fourier space as [18]

$$
\mathrm{SNR}^{2}=\iint \frac{|\Delta G(u)|^{2}}{\operatorname{NPS}(u)} d^{2} u,
$$

where $\Delta G$ is the expected signal difference between object and background, and NPS is the noise power spectrum for the background. Images from simulations were used to obtain the noise-free signals.

For PBI, the simulation starts with a plane wave, using the projection approximation to obtain the wavefield after the object, then Fresnel propagation to calculate the wavefield at the effective propagation distance. The wavefield is converted into detector response taking account of the point spread function (PSF), the quantum efficiency and the energy response of the detector. Photon noise and read-out noise are considered as noise sources in the simulation. For GBI, the basic procedure is the same as PBI, but with the two gratings and the scanning process added [14, 16]. More complicated simulations are required for accurate scattering information, but for our PET sample the scattering is small and not considered in this study.

\section{RESULTS AND DISCUSSIONS}

\subsection{Simulation verification}
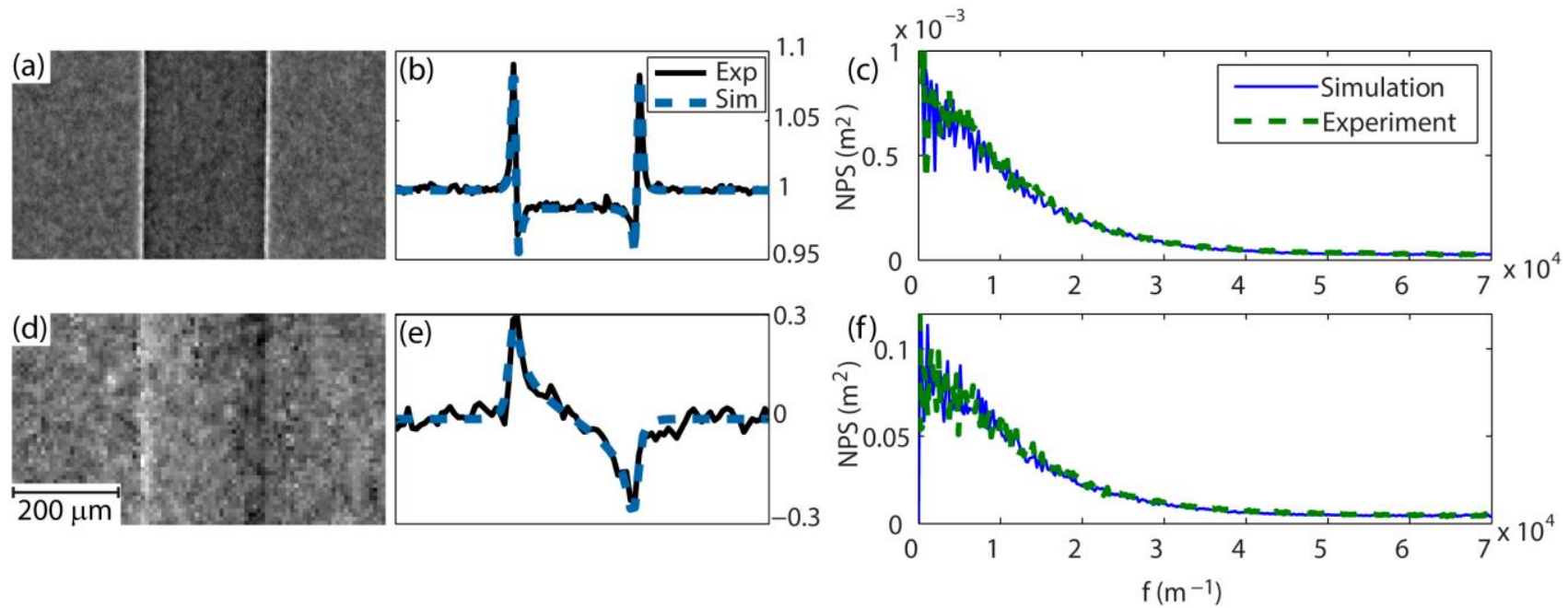

Figure 2. PBI projection (a, b) and DPC image (c, d) of a polyethylene terephthalate (PET) cylindrical phantom in a liquidparaffin bath. Projections (a, d) have 4 hours total exposure time and 0.7 Gy radiation dose. The solid lines in (b) and (c) are profiles of the experimental image (a) and (d), respectively, averaged longitudinally for better visuality. The dashed lines are profiles from simulations without noise. (c, f) NPS of the background from experiment and simulation for PBI and GBI, respectively.

Experimental images of a PET phantom with a diameter of $213 \mu \mathrm{m}$ for the two methods with the same total exposure time show that the PBI image has lower noise level than the GBI differential phase contrast (DPC) image as shown in Figure 2. Simulations show good agreement with experiment as found in the profiles (b, e) and the NPS (c, f).

\subsection{Detectability comparison}

Detectability of the PET phantom for projection images is studied as a relation between the required radiation dose for detection and the phantom diameter. The required dose is calculated when $\mathrm{SNR}^{2}=25$ as the limit of whether an object is considered as observable [19]. To avoid the effect of the phantom length on detectability, the images used to calculate $\mathrm{SNR}^{2}$ are assumed to have a height the same as the phantom diameter. For both methods the doses are calculated with 
the mass energy-absorption coefficients from NIST [20]. The radiation doses have been compared with Monte Carlo simulations using the Penelope 2006 software [21] and differences of at most $23 \%$ were found. As the doses of the two methods listed here are for comparison, and differ by an order of magnitude for many investigated object diameters, this error is considered small.

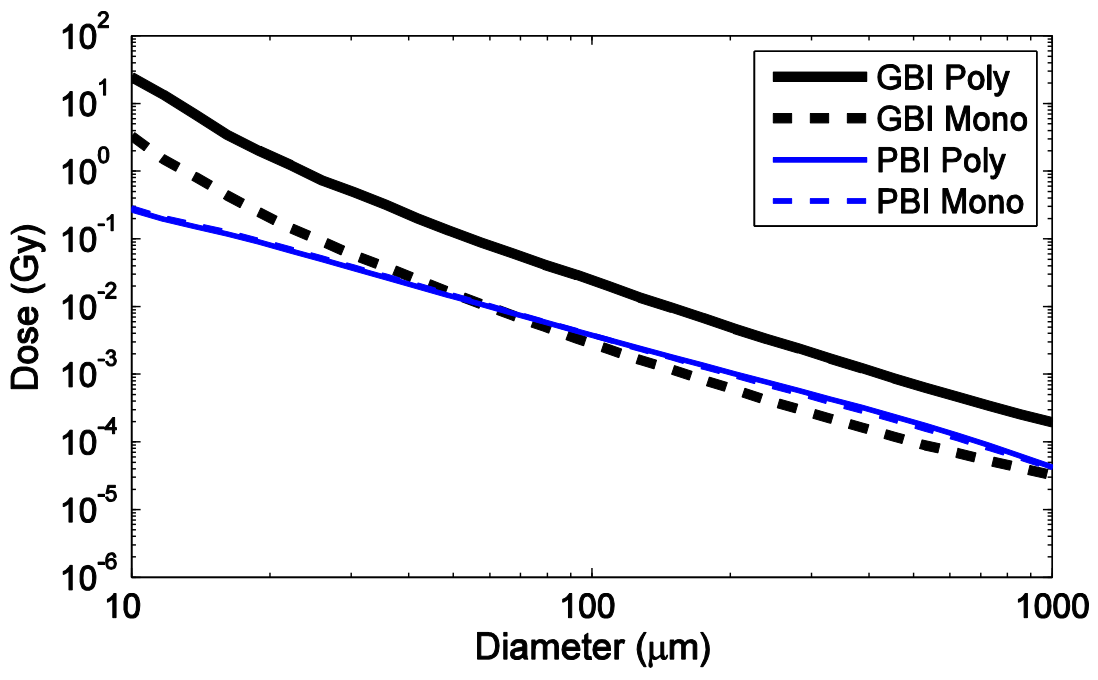

Figure 3. Doses required for detecting a simulated PET cylinder as a function of the cylinder diameter. Poly lines assume a liquid-metal-jet source spectrum and Mono lines $25 \mathrm{keV}$ monochromatic x-rays.

As shown in Figure 3, using a polychromatic source, the required dose for PBI is lower than for GBI, and the exposure time shorter. Using a monochromatic source, PBI requires lower doses and shorter exposure time for smaller-diameter phantoms while GBI performs better for larger-diameter phantoms. This result agrees with the predictions from Diemoz et al [9] and can be understood as the inherent difference of the two methods. PBI measures the second derivative of the phase while GBI measures the first. The contrast transfer functions of them are quadratic and linear, respectively, which indicates that GBI favors lower spatial resolution more than PBI. All the simulations are done with the assumption of a pair of gratings without defects, which have $50 \%$ duty cycle, correct periods and correct phase shift. With realistic gratings which lead to a decrease to the visibility of the images, the dose and exposure time for GBI will increase correspondingly.

The simulations in Figure 3 all assume unbinned images with a detector pixel size of $9 \mu \mathrm{m} \times 9 \mu \mathrm{m}$. In Figure 4 simulations are done with different binnings assuming a monochromatic source, while detector PSF and other parameters are all kept unchanged. The $2 \times 2$ and $6 \times 6$ binning produce pixel sizes of 18 and $54 \mu \mathrm{m}^{2}$, respectively. As the binned system cannot resolve the finest features shown in Figure 3, those are excluded from Figure 4 and the scale instead starts at $50 \mu \mathrm{m}$. It can be seen that PBI suffers more than GBI when increasing the pixel size since PBI requires small pixel size to resolve the edge enhancements that contain phase information. Thus in applications where high resolution is not required, a lower resolution but more efficient detector can be used for GBI. Also a higher power extended source together with a source grating can be used to reduce the exposure time. 


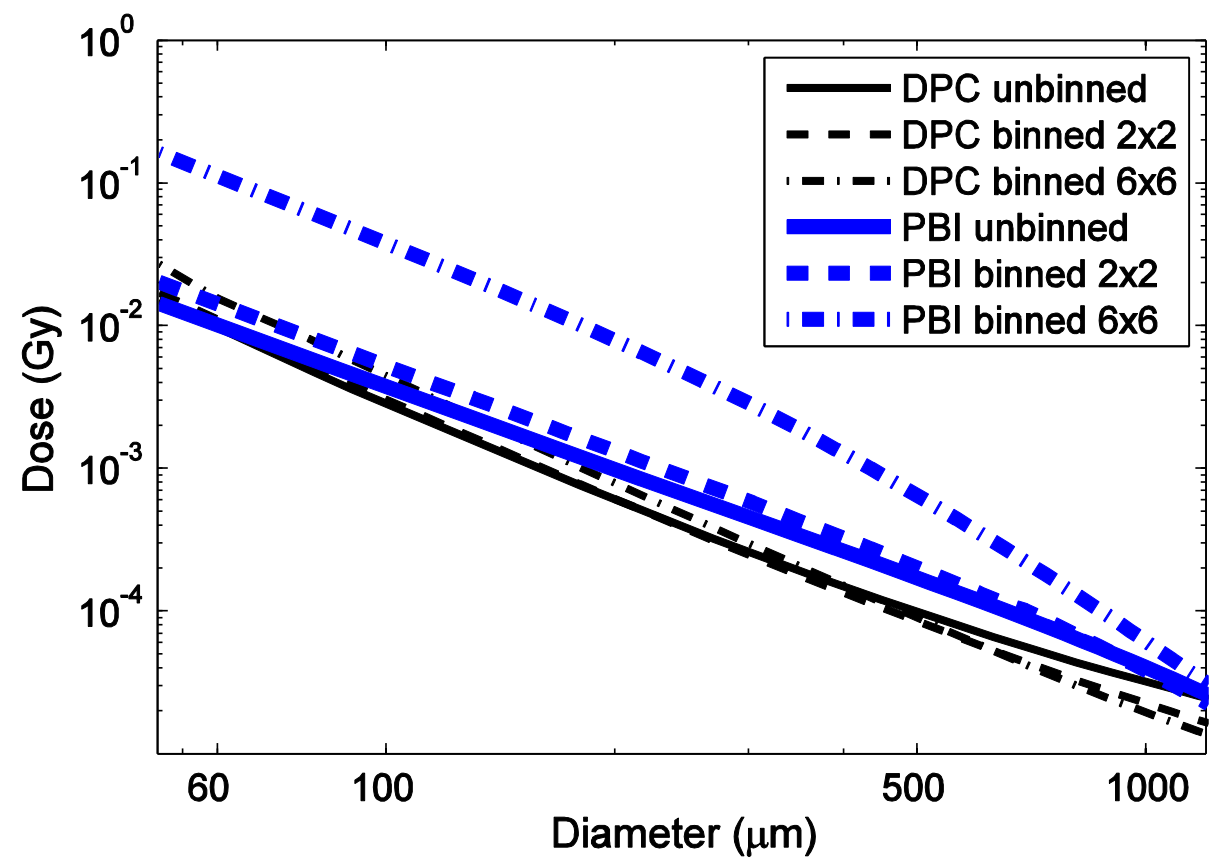

Figure 4. Simulations assuming monochromatic source with different binnings of the detector response.

\subsection{Biological applications}

Some biological samples were scanned with the same experimental arrangements in order to make a qualitative comparison. Figure 5(a) is a reconstructed slice from a PBI tomogram of a rat brain. The exposure time is in total $60 \mathrm{~h}$ for a tomogram with 720 projections over 360 degrees. The reconstruction is done with filtered back projection (FBP) in the Octopus software [22] after phase retrieval with the parameters of brain tissue and paraffin oil [20, 23]. The phase retrieval is Fourier-based assuming a homogeneous sample to reconstruct the projected density [24]. Figure 5(b) shows a region of hippocampus and (c) a region with cerebral aqueduct. Figure 5(d-f) shows the same object with the same total exposure time and the same amount of flatfield images for DPC CT. The reconstruction is done with FBP on Hilbert filtered DPC images [25]. Compared to (a-c), (d-f) are noisier, besides which, the cytoarchitectural boundaries in hippocampus shown in (b) are hardly visible in (e). 


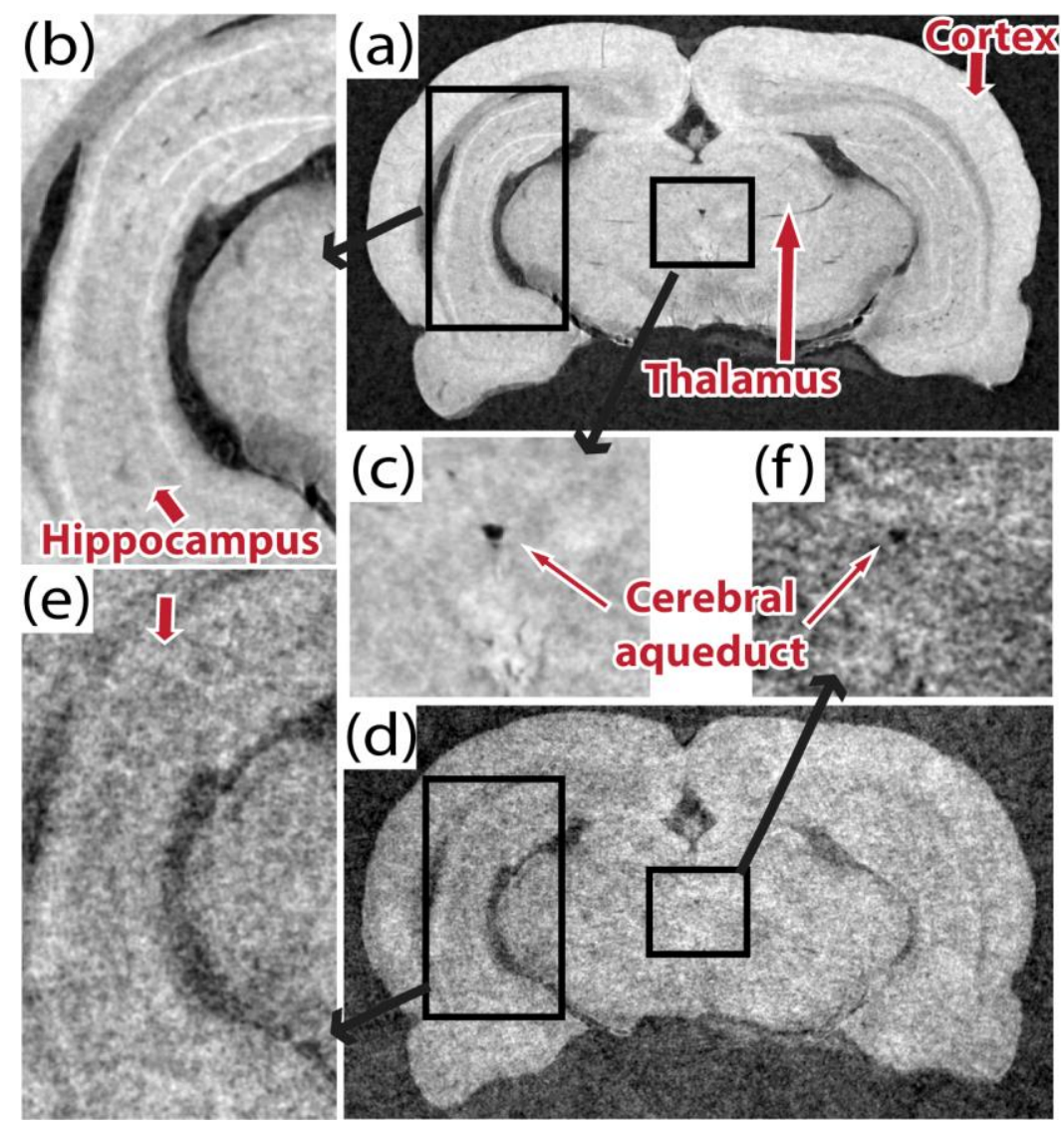

Figure 5. Rat brain reconstruction slice of (a-c) PBI and (d-f) GBI phase image. (b,e) are the magnified image of a region of hippocampus and $(\mathrm{c}, \mathrm{f})$ a region containing cerebral aqueduct. The PBI and GBI tomograms had the same total exposure time.

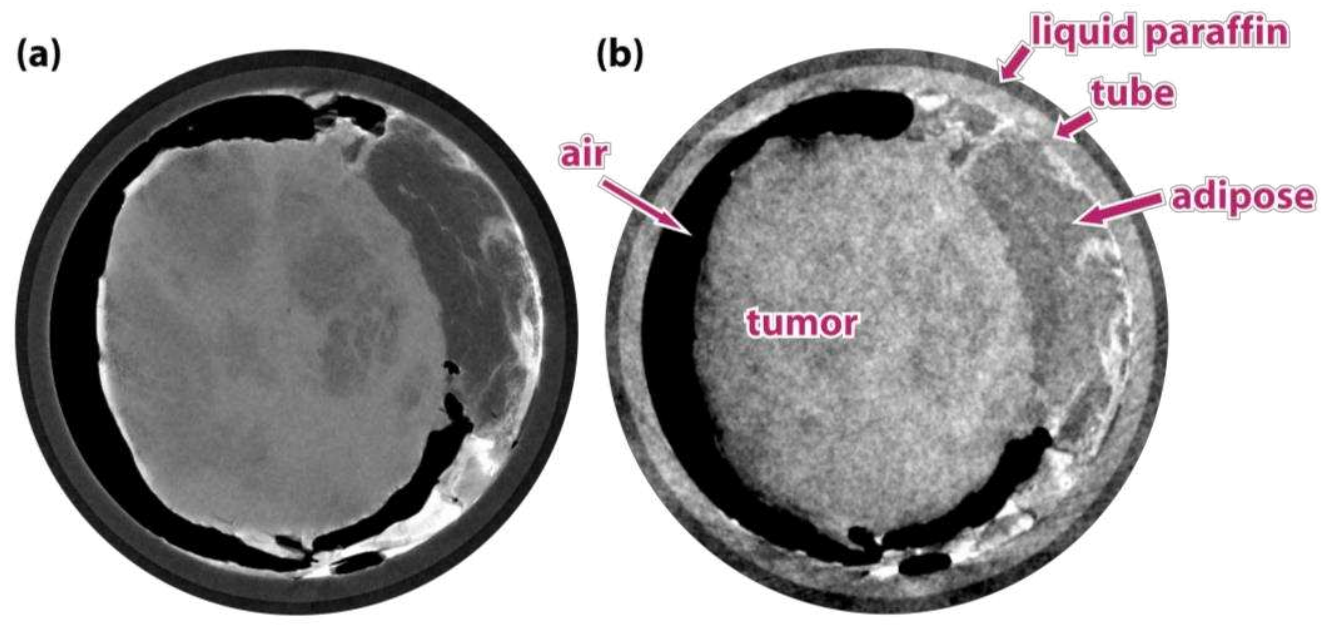

Figure 6. Breast biopsy sample phase CT slice using (a) PBI and (b) GBI.

Figure 6 shows experimental tomographic slices of a breast biopsy sample's phase information using both PBI and GBI with the same exposure time and dose as the scan in Figure 5. The phase retrieval for single-distance PBI is done with the parameters of adipose tissue and breast tissue, therefore the interfaces between other materials may not be phaseretrieved correctly. For example, there is still edge enhancement between the air and the breast tissue as shown in Figure 
6(a). For GBI, phase information of all materials can be retrieved simultaneously without need of pre-knowledge of the object.

\section{CONCLUSIONS}

We compare the detectability of PBI and GBI with respect to dose. Experiments are done with a liquid-metal-jet source intended for applications in laboratory systems. Simulations done with projection approximations show good agreement with experiments. The results show that the polychromaticity of the source affects GBI a lot more than PBI. In the studied object size range $(10-1000 \mu \mathrm{m})$, PBI shows better detectability than GBI when using a polychromatic source even if assuming a pair of gratings without defects. Using a monochromatic source, PBI performs better for small object sizes while GBI performs better for larger ones. GBI shows better compatibility for larger pixel sizes than PBI. We show some 3D biological applications of the two methods with the same exposure time and dose for comparison. PBI gives higher SNR, but GBI has the advantage of proper phase-retrieval for multi-material objects.

\section{ACKNOWLEDGEMENTS}

The authors would like to thank Karlsruhe Institute of Technology for providing the gratings, Zhentian Wang for providing the breast biopsy sample, Anders Holmberg for the support on phase-stepping mechanics. Financial support was received from the Swedish Research Council, the Wallenberg Foundation, and the Göran Gustafsson foundation.

\section{REFERENCES}

[1] S. W. Wilkins, T. E. Gureyev, D. Gao et al., "Phase-contrast imaging using polychromatic hard X-rays," Nature, 384(6607), 335-338 (1996).

[2] T. Weitkamp, A. Diaz, C. David et al., "X-ray phase imaging with a grating interferometer," Opt. Express, 13(16), 6296-6304 (2005).

[3] F. Pfeiffer, T. Weitkamp, O. Bunk et al., "Phase retrieval and differential phase-contrast imaging with low-brilliance X-ray sources," Nat. Phys., 2, (2006).

[4] F. Pfeiffer, M. Bech, O. Bunk et al., "Hard-X-ray dark-field imaging using a grating interferometer," Nat. Mater., 7(2), 134-137 (2008).

[5] T. Kohler, K. Jurgen Engel, and E. Roessl, "Noise properties of grating-based x-ray phase contrast computed tomography," Med. Phys., 38, S106-S116 (2011).

[6] R. Raupach, and T. G. Flohr, "Analytical evaluation of the signal and noise propagation in x-ray differential phasecontrast computed tomography," Phys. Med. Biol., 56(7), 2219-2244 (2011).

[7] D. Hahn, P. Thibault, M. Bech et al., "Numerical comparison of X-ray differential phase contrast and attenuation contrast," Biomed. Opt. Express, 3(6), 1141-1148 (2012).

[8] X. Tang, Y. Yang, and S. Tang, "Characterization of imaging performance in differential phase contrast CT compared with the conventional CT--noise power spectrum NPS(k)," Med. Phys., 38(7), 4386-4395 (2011).

[9] P. C. Diemoz, A. Bravin, M. Langer et al., "Analytical and experimental determination of signal-to-noise ratio and figure of merit in three phase-contrast imaging techniques," Opt. Express, 20(25), 27670-27690 (2012).

[10]P. C. Diemoz, A. Bravin, and P. Coan, "Theoretical comparison of three X-ray phase-contrast imaging techniques: propagation-based imaging, analyzer-based imaging and grating interferometry," Opt. Express, 20(3), 2789-2805 (2012).

[11]V. Revol, C. Kottler, R. Kaufmann et al., "Noise analysis of grating-based x-ray differential phase contrast imaging," Rev. Sci. Instrum., 81, 073709 (2010).

[12]G. H. Chen, J. Zambelli, K. Li et al., "Scaling law for noise variance and spatial resolution in differential phase contrast computed tomography," Med. Phys., 38(2), 584-588 (2011).

[13]K. Li, N. Bevins, J. Zambelli et al., "Fundamental relationship between the noise properties of grating-based differential phase contrast CT and absorption CT: theoretical framework using a cascaded system model and experimental validation," Med. Phys., 40(2), 021908-021922 (2013).

[14]U. Lundström, D. H. Larsson, A. Burvall et al., "X-ray phase contrast for $\mathrm{CO}_{2}$ microangiography," Phys. Med. Biol., 57(9), 2603-2617 (2012).

[15]R. Raupach, and T. Flohr, "Performance evaluation of x-ray differential phase contrast computed tomography (PCT) with respect to medical imaging," Med. Phys., 39(8), 4761-4774 (2012). 
[16]T. Zhou, U. Lundström, T. Thüring et al., "Comparison of two x-ray phase-contrast imaging methods with a microfocus source," Opt. Express, 21(25), 30183-30195 (2013).

[17]T. Thüring, T. Zhou, U. Lundström et al., "X-ray grating interferometry with a liquid-metal-jet source,” Appl. Phys. Lett., 103(9), 091105 (2013).

[18]H. H. Barrett, and K. J. Myers, [Foundations of Image Science] John Wiley \& Sons, Inc, (2003).

[19]A. Rose, "The sensitivity performance of the human eye on an absolute scale," J. Opt. Soc. Am., 38(2), 196-208 (1948).

[20]J. H. Hubbell, and S. M. Seltzer, [Tables of X-Ray Mass Attenuation Coefficients and Mass Energy-Absorption Coefficients from $1 \mathrm{keV}$ to $20 \mathrm{MeV}$ for Elements $\mathrm{Z}=1$ to 92 and 48 Additional Substances of Dosimetric Interest] Radiation and Biomolecular Physics Division, PML, NIST, (1996).

[21]J. Baro, J. Sempau, J. M. Fernandezvarea et al., "Penelope - an Algorithm for Monte-Carlo Simulation of the Penetration and Energy-Loss of Electrons and Positrons in Matter," Nucl. Instr. Meth. Phys. Res. B, 100(1), 31-46 (1995).

[22]J. Vlassenbroeck, M. Dierick, B. Masschaele et al., "Software tools for quantification of X-ray microtomography," Nucl. Instr. Meth. Phys. Res. A, 580(1), 442-445 (2007).

[23]L. Kissel, "RTAB: the Rayleigh scattering database," Radiat. Phys. Chem., 59(2), 185-200 (2000).

[24]D. Paganin, S. C. Mayo, T. E. Gureyev et al., "Simultaneous phase and amplitude extraction from a single defocused image of a homogeneous object," J. Microsc., 206(Pt 1), 33-40 (2002).

[25]F. Pfeiffer, C. Kottler, O. Bunk et al., “Hard x-ray phase tomography with low-brilliance sources,” Phys Rev Lett, 98(10), 108105-108108 (2007). 